Archives

$5 \mid 1990$

Varia

\title{
Les justices de l'au-delà. Les représentations de l'enfer en France et en Italie ( $\mathrm{XII}^{\mathrm{e}}-\mathrm{XV}^{\mathrm{e}}$ siècles)
}

\author{
Jérôme Baschet
}

\section{(2) OpenEdition Journals}

\section{Édition électronique}

URL : http://journals.openedition.org/ccrh/2886

DOl : $10.4000 /$ ccrh.2886

ISSN : $1760-7906$

Éditeur

Centre de recherches historiques - EHESS

Édition imprimée

Date de publication : 15 avril 1990

ISSN : 0990-9141

\section{Référence électronique}

Jérôme Baschet, « Les justices de l'au-delà. Les représentations de l'enfer en France et en Italie (xII e-xv e siècles) ", Les Cahiers du Centre de Recherches Historiques [En ligne], 5| 1990, mis en ligne le 20 mars 2009, consulté le 19 avril 2019. URL : http://journals.openedition.org/ccrh/2886 ; DOI : 10.4000/ ccrh.2886

Ce document a été généré automatiquement le 19 avril 2019

Article L.111-1 du Code de la propriété intellectuelle. 


\title{
Les justices de l'au-delà. Les représentations de l'enfer en France et en Italie ( $\mathrm{XII}^{\mathrm{e}}-\mathrm{XV}^{\mathrm{e}}$ siècles)
}

\author{
Jérôme Baschet
}

\section{NOTE DE L'AUTEUR}

Ce texte reprend les éléments développés dans une thèse de doctorat soutenue à l'École des Hautes Études en Sciences Sociales en janvier 1989, et en cours de publication dans la Collection de l'École française de Rome. Cette recherche a été menée sous la direction de Jacques Le Goff et avec le constant soutien de Jean-Claude Schmitt et Jean-Claude Bonne.

1 À la rencontre de l'imaginaire des supplices et de la conception chrétienne du péché, l'enfer médiéval est le lieu où résonnent les plaintes de l'homme coupable voué à un châtiment sans fin. Il est au coeur du système chrétien qui promet dans l'autre monde une rétribution - en bien ou en mal - des actes accomplis ici-bas. Doté d'une présence massive, incontournable, l'enfer n'est assurément pas le domaine des demi-mesures : il participe de la dimension duelle du christianisme médiéval, dans lequel le purgatoire, à la fin du XII ${ }^{\mathrm{e}}$ siècle, introduit un peu de souplesse.

2 Pourtant, alors même que l'enfer apparaît comme un élément central dans la religion médiévale et plus largement dans les mentalités et la vie, sociale, il paraît résister à l'histoire. Tout le problème, précisément, est d'en faire un objet d'histoire. Lorsque, au terme du chapitre sur La société féodale consacré aux "façons de sentir et de penser ", Marc Bloch se demande «comment ne pas reconnaître dans la peur de l'enfer un des grands faits sociaux du temps ", il souligne le rôle d'une telle croyance dans la société, au sein d'un monde où le religieux et le politique ne cessent d'afficher les formes de leur imbrication. Et le problème se complique encore si, à l'énoncé de la fonction historique de la croyance, on ajoute une interrogation sur son importance. Car plutôt que de tenir la peur de l'enfer pour une évidence, il importance. Car plutôt que de tenir la peur de l'enfer 
pour une évidence, il convient d'analyser son fonctionnement, de s'interroger sur ses limites et en particulier sur les rapports entre la doctrine chrétienne de l'au-delà et les conceptions plus diversifiées que l'on peut repérer au Moyen Age, des croyances folkloriques aux évocations d'un enfer carnavalesque cher à M. Bakhtine.

En outre, l'importance du thème infernal ne peut être tenue pour constante au cours des quatre siècles considérés, d'autant plus que le problème de la chronologie recoupe celui de l'interprétation des derniers siècles du Moyen Age et en particulier du thème de la mort. Faut-il voir alors, avec A. Tenenti, le « chant du cygne » de l'iconographie infernale et décrire un remplacement de la crainte de l'enfer par un souci de la mort, signe d'un attachement à la vie terrestre et d'une première rupture avec le christianisme dominant du Moyen-Age, ou du moins, avec P. Ariès, un effacement de la perspective eschatologique au profit d'une préoccupation de la mort de soi ? Ou peindre au contraire, avec J. Huizinga, M. Vovelle et J.Delumeau, un Moyen Age finissant aux couleurs automnales, sombrant dans le pessimisme et voyant s'affirmer un «christianisme de la peur » dans lequel l'enfer et son image tiennent la vedette. Pour affronter ce problème, il faudra proposer des critères de mesure pertinents, ce qui suppose de ne pas considérer l'enfer comme un élément isolé, mais d'analyser au contraire l'ensemble du système au sein duquel il fonctionne.

4 Mais l'enfer n'est pas seulement un rouage du système religieux et social ; c'est aussi un paysage où prend corps tout un champ de l'imaginaire: domaine infini des ténèbres et d'un feu qui n'éclaire pas, grouillant de répugnants reptiles et de monstres dévorants, où le corps des damnés est soumis à toutes les tortures. L'enfer apparaît comme un lieu privilégié d'expression d'un imaginaire négatif, tissé des peurs, des angoisses, des rejets de l'homme. Y a-t-il là un objet d'étude pertinent? Oui, à condition de prêter aux descriptions et aux représentations assez d'attention pour ne pas y voir seulement l'inlassable répétition de motifs stéréotypés. On peut alors analyser la cohérence et la richesse symbolique de ces représentations, comme l'a fait G. Le Don en utilisant les catégories définies par G. Durand. On peut aussi, sans nier l'existence d'un socle relativement stable, s'attacher à repérer les transformations qui affectent les mises en forme de cet imaginaire, et la diversité des modèles qui se succèdent ou coexistent dans le temps.

5 Pour saisir une réalité historique complexe dans la variété de ses formes et de ses fonctions, il fallait interroger des sources aussi diversifiées que possible, de la théologie aux récits de visions, de la littérature morale au théâtre religieux, des miniatures à l'art monumental, fresques, mosaïques ou sculptures. Mais c'est essentiellement autour des images que s'organise la démarche, ce qui impose un infléchissement de la perspective. Car il serait dangereux de faire un usage pressé des images, qui consisterait pour l'historien à les soumettre d'emblée à ses questions (à la question). Non qu'on croit à une quelconque anhistoricité des phénomènes iconographiques: il est clair qu'ils sont l'expression d'une pensée et d'une vision du monde propres à une civilisation et à un temps donnés. Mais ils les expriment à leur manière, avec leur règles propres (et dont l'historicité n'est pas nécessairement réductible, sans autre forme de procès, à l'Histoire tout court). L'historien doit donc se faire " analyste d'images ", s'attacher à en saisir les modes de fonctionnement, à comprendre la structure d'ensemble des images au sein desquelles l'enfer n'est qu'un élément, sans négliger la fonction du support ou l'organisation du lieu où apparaît sa représentation. L'usage des images impose donc de longs détours, qui sont la marque du respect dû à la spécificité de la pensée figurative. 
6 Cette recherche a donc été menée dans une double optique, visant à la fois à rendre compte de la richesse d'une production imaginaire souvent luxuriante et à analyser les fonctions qu'assume la thématique infernale au sein d'un système religieux et social complexe. A l'évidence, on ne pourra aborder, dans le cadre de cette présentation, les questions soulevées par tous les types de sources considérés, ni rendre pleinement justice à la fécondité des thèmes mis en oeuvre par les textes et les images infernales. Aussi s'attachera-t-on principalement à dessiner l'évolution des représentations infernales, dont l'histoire peut s'organiser autour des fresques du Camposanto de Pise (1330-1340), véritable point de fuite de l'enquête. Puis, on évoquera en finissant quelques problèmes généraux touchant au fonctionnement de l'image infernale, à ses effets et aux échos qu'elle suscite.

\section{Les origines du châtiment éternel}

Dans l'histoire des religions, l'idée d'une rétribution post mortem des mérites et des démérites émerge lentement. Elle ne joue guère de rôle dans les civilisations anciennes de la Méditerranée, qui, dans un premier temps, conçoivent un monde des morts souterrain et largement indifférencié (l'Hadès des Grecs ou le Shéol des Juifs). Dans le judaïsme, la justice de Yahvé s'exerce sur terre, pour récompenser ou pour punir, et c'est seulement dans les livres les plus tardifs de l'Ancien Testament que la perspective de la réalisation messianique du Royaume tend à glisser du domaine terrestre vers l'autre monde. Enfin, dans la littérature apocalyptique, en particulier dans le Livre d'Hénoch (II ${ }^{\mathrm{e}} \mathrm{s}$. av. J.-C.), s'affirme nettement la perspective d'un grand Jugement des morts, venant compenser les injustices subies sur terre.

Mais c'est au christianisme qu'il revenait de porter cette mutation à son terme, en plaçant au centre du système religieux le principe d'une rétribution et d'un châtiment après la mort. Le message évangélique souligne la stricte bipartition de l'au-delà - enfer pour les uns, paradis pour les autres - dont l'importance est encore renforcée par le rôle du Jugement dernier et de cet élément fondamental qu'est la résurrection des corps (Mt. 24-25 ; Ap. 20-21). Dès lors, ce qu'on peut appeler le principe d'inversion, jouant d'un retournement complet et sans appel entre le sort terrestre et le sort dans l'au-delà, est un caractère fondamental du christianisme : qui a vécu dans le plaisir sera dans la peine ; qui a vécu dans la peine connaîtra la béatitude.

Pourtant, cette idée ne s'affirme pas sans mal, même dans le monde chrétien, comme si l'on avait du mal à tailler dans le vif de la Création et à exclure de la miséricorde divine une partie entière de l'humanité. Témoins de ces hésitations : Origène, auquel on a prêté, en forçant peut-être sa pensée, la doctrine d'une réunion finale de toutes les créatures à Dieu (apocatastase) et Augustin qui, affirmant avec netteté l'éternité des peines, doit consacrer un livre entier de la Cité de Dieu à réfuter les objections que soulève cette idée. Après Augustin, l'idée de l'éternité est théologiquement incontestée, mais il n'est pas sûr qu'elle ait pour autant pénétré tous les esprits et qu'elle ait pris toute l'importance qui lui revient en principe. De fait, l'évolution au cours du Moyen Age peut apparaître comme la réalisation effective, dans les mentalités et dans la société, de l'idée d'une rétribution post mortem fondée sur le principe de l'inversion. 


\section{Dans les écrits cléricaux : de la théologie aux récits de visions}

10 Pour les théologiens des $\mathrm{XII}^{\mathrm{e}}$-XIII ${ }^{\mathrm{e}}$ siècles, de Hugues de Saint-Victor à Saint-Thomas, l'enfer ne fait plus l'objet de controverses fondamentales; il s'agit surtout de préciser le tableau de la condition des damnés : le plus terrible est la peine du dant - le fait d'être séparé de Dieu - même si la peine du sens - essentiellement le feu, le froid, les ténèbres et les vers - n'est aucunement négligée. De fait, les théologiens ne cherchent pas à écarter la dimension corporelle, physique des châtiments et, même s'il ne leur revient pas d'en développer la description, on doit souligner que saint Thomas admet le principe d'une diversité des peines corporelles.

11 La seule question délicate concerne le sort des âmes entre la mort individuelle et le Jugement : peuvent-elles aller directement dans ces lieux matériels que sont l'enfer et le paradis ou, considérant qu'un être spirituel ne peut être retenu en un lieu matériel et, moins encore, tourmenté par un feu corporel, doit-on croire qu'elles attendent le Jugement dans des lieux provisoires, plus conformes à leur nature? Derrière son apparente futilité, le problème engage une question anthropologique fondamentale, celle des relations entre le corps et l'âme, entre le corporel et le spirituel. Elle suppose également de clarifier le rapport entre le Jugement dernier et le jugement qui survient lors de la mort - rapport qui, aux XII-XIII ${ }^{\mathrm{e}}$ siècles, est de complémentarité et en aucun cas de substitution. Après une période où coexistent deux traditions, l'une spiritualiste, l'autre corporéïste, la conception des lieux d'attente est très largement abandonnée dans la seconde moitié du XII ${ }^{\mathrm{e}}$ siècle, dans le cadre de la vaste réorganisation de l'au-delà qui s'opère alors et dont la naissance du purgatoire comme lieu est un des signes les plus marquants (le purgatoire se constitue comme lieu spécifique au moment où on affirme que les âmes vont directement en enfer ou au paradis, et non plus dans des lieux d'attente).

Dans les traités moraux, les évocations de l'enfer se simplifient et se concentrent sur la nature des peines, comme dans l'Elucidarium d'Honorius Augustodunensis dont la description des neuf peines d'enfer eut une influence considérable. Les exempla illustrent, au sein d'une pratique renouvelée de la prédication, le développement d'une rhétorique morale efficace, qui fait jouer toutes les figures possibles du principe d'inversion. Ils intègrent des descriptions de plus en plus développées de peines multiples, frappantes, généralement en rapport avec la nature de la faute punie. En ce sens, ils participent au renforcement de la matérialité et de l'immédiateté des châtiments, ce qu'on ne peut expliquer uniquement par la nécessité de convaincre un public réputé vulgaire. En effet, il n'y a pas lieu d'opposer une vision concrète de l'enfer, nécessairement « populaire », et une conception savante, répugnant à considérer la matérialité des châtiments.

$13[. . .]^{1}$ Sans parler nécessairement d'anticipation, on doit considérer cette oeuvre comme une « anomalie historique ", du moins au regard de la chronologie, même si son existence peut être rattachée à un contexte très particulier de forte conflictualité, et mise en rapport avec les oeuvres suscitées en Italie centrale par les tensions liées à la Réforme grégorienne.

14 A l'époque gothique, le Jugement dernier se développe fortement, hissant l'enfer avec lui au portail de nombreuses cathédrales. Toutefois, l'image de l'enfer reste limitée: la 
représentation théophanique se concentre alors sur le moment du Jugement, sur la scène et les opérations du partage, de sorte que l'enfer apparaît marginal, évoqué comme destination du cortège des damnés. Dans le domaine français, une représentation de l'enfer s'impose alors tant dans le Jugement dernier que dans les autres contextes thématiques, comme la Chute des anges, la Descente aux limbes, la Parabole de Lazare, les scènes de l'Apocalypse, ou l'illustration de textes littéraires : la gueule de Léviathan ouvre ses crocs pour dévorer les pécheurs.

La gueule connaît une variété formelle stupéfiante, jouant parfois sur l'association de gueules multiples; elle suggère une agressivité dévorante comme dans l'image du Psautier de Winchester, ou insiste sur un dynamisme sinueux, digestif, comme dans les miniatures des Apocalypses anglo-normandes du XIII ${ }^{e}$ siècle. Elle tourne vers le spectateur ou le lecteur les formes acérées de ses crocs, cherche à le capturer par la force de fascination de son regard. Ailleurs au contraire, le motif est dépourvu de toute tension et peut même perdre tout pouvoir d'effroi. De fait, la gueule, figuration quasi obligée de l'enfer, oscille entre un déploiement flamboyant de l'horreur et des emplois répétés mécaniquement (comme dans les Bibles moralisées), entre la puissance d'une grandiose métaphore et le recours à un motif conventionnel qui fait d'elle un simple signe, utilisé moins pour sa force expressive que pour sa valeur de reconnaissance (elle permet alors d'identifier comme infernal le lieu où se déroule la scène représentée).

Il est surprenant de constater qu'en Italie, comme à Byzance dont son art s'inspire d'abord, la gueule n'apparaît quasiment pas. En fait, c'est toute la composition (et à travers elle la conception) du Jugement dernier qui diffère ici. Pour s'en tenir à l'enfer, notons que celui-ci, au lieu de figurer seulement comme destination du cortège des damnés, apparaît comme un lieu spécifique, délimité par un cadre propre, et centré sur la figure trônante de Satan (San Angelo in Formis, Baptistère de Florence, panneau de Grosseto). Et même si, à l'exception du Jugement de Torcello (fin xie siècle), la figuration de l'enfer ne donne lieu à aucune image structurée des peines, il est important qu'existe déjà le cadre d'une telle représentation. Avec le Jugement peint par Giotto à Padoue (c. 1304), qui intègre un important développement des supplices, une nouvelle étape est franchie. Toutefois, les scènes sont disposées sur le fond noir de l'enfer, sans architecture d'ensemble, tandis que la petite taille des damnés suggère une présence ambiguë, comme si les supplices n'avaient pas encore achevé le chemin qui devait les conduire sur le devant de la scène.

\section{Au campo Santo de pise : la naissance d'un système pénal}

Dans les années 1330-1340, Buonamico Buffalmaco peint, sur les murs du cimetière monumental de Pise, un cycle de fresques associant le Jugement dernier, l'Enfer, le Triomphe de la Mort et la Thébaïde. Celui-ci combine un ensemble de nouveautés et de mutations si considérable qu'on est tenté de parler de révolution iconographique. Le développement des supplices rejoint ici le degré de maturation constaté dans les visions du XII siècle: le corpus des châtiments s'étend, les modes d'attaque du corps se diversifient, incluant en particulier des formes de rupture de l'intégrité du corps (décapitation, amputations, écorchage). La visée du corps, découpé, violé, atteint un paroxysme de violence. 

représentation est désormais compartimentée par une structure rocheuse et par la figure centrale de Satan. Cette division correspond à une logique du châtiment, puisque chaque lieu (ou presque) est spécifiquement consacré à la punition d'un péché capital. L'utilisation dominante du septénaire des péchés constitue une mutation essentielle : non seulement elle accompagne la mise en place d'un ordre du châtiment, mais elle contribue, par le recours à un modèle qui englobe l'ensemble des fautes possibles, au recul de l'infigurabilité de l'enfer. De fait, Buffalmaco s'emploie à suggérer que l'enfer est montré dans sa totalité; son œuvre se donne comme une figuration complète du royaume de Satan. qu'elles punissent. Sans être nouveau, ce principe connaît ici une application généralisée. Ainsi, voit-on les avares gavés de pièces d'or fondues, les gloutons placés autour d'une table garnie sans pouvoir rien manger, les hérétiques décapités et les schismatiques amputés pour avoir divisé le corps de l'Église, les sodomites empalés et réunis en une éternelle union, ou les coupables d'accédie (paresse-mélancolie) représentés dans une attitude de prostration qui ne fait que prolonger leur trouble mental. Dès lors, un rapport de signifiant à signifié s'établit entre le supplice et la faute, selon des modalités variables permettant une lecture plus ou moins évidente. L'image exige une conversion du spectacle de l'horreur en leçon morale.

La fresque de Pise transforme également le rapport entre Jugement dernier et enfer : au lieu d'être confinée dans un angle de l'image du Jugement, la caverne infernale est placée à côté du Jugement et occupe presque autant de place que lui. Il s'agit ainsi d'une image bipolaire du Jugement, dont une moitié est centrée sur la figure de Satan et l'autre sur le Christ-Juge ; et le rapport est d'autant plus troublant que l'affirmation du pôle infernal (Satan est environ deux fois plus grand que le Christ) va de pair avec une division du pôle positif, le Christ partageant à égalité avec la Vierge la fonction judiciaire (cas unique dans l'art monumental). Et même s'il ne s'agit pas à proprement parler de deux images séparées, l'enfer bénéficie d'un extraordinaire processus d'autonomisation, qui constitue un critère, qualitatif et non uniquement quantitatif, permettant de mesurer son affirmation.

21 Au total, l'importance des mutations enregistrées à Pise autorise à considérer cette oeuvre comme une césure fondamentale, d'autant plus qu'elle inaugure un modèle largement repris ensuite dans la peinture italienne. Certes, on doit rappeler le précédent que constitue, sur certains points, l'oeuvre de Giotto, et noter que le travail de Buffalmaco s'inscrit aussi dans une dynamique propre au schéma italien du Jugement dernier qui, faisant prévaloir une dualité entre l'enfer et le Christ-Juge, plutôt qu'entre l'enfer et un lieu paradisiaque placé en regard, tendait à repousser l'enfer hors de l'image du Jugement.

22 On ne peut pas non plus ignorer l'Enfer de Dante (1309-1313), qui partage avec la fresque de Buffalmaco des principes fondamentaux: ordonnancement très poussé de l'enfer, division en différents lieux, classification rigoureuse des catégories pénales, affirmation d'une révélation intégrale de l'enfer. Quelques motifs ponctuels montrent en outre que l'oeuvre de Dante était connue dans le cercle des concepteurs de la fresque. Pourtant, ni la structure générale, ni le découpage des catégories pénales, ni la nature des peines utilisées dans les deux oeuvres ne sont véritablement comparables. En aucun cas, la fresque de Buffalmaco ne peut être considérée comme une mise en image de l'Enfer de 
Dante, et les deux oeuvres apparaissent comme des applications parallèles de principes comparables, mais dont les intentions sont par ailleurs largement différentes.

Afin de comprendre la dimension morale et didactique des fresques, c'est moins vers des « sources » que vers le contexte pisan qu'il convient de se tourner. Haut lieu de la culture dominicaine, le couvent dominicain de Santa Caterina, principal commanditaire du cycle, s'est rendu célèbre grâce à des hommes comme Giordano da Pisa, figure marquante de la prédication italienne dans les premières années $d u \mathrm{XIV}^{\mathrm{e}}$ siècle, ou, à l'époque même de Buffalmaco, comme Domenico Cavalca, vulgarisateur infatigable, notamment des Vies des Pères, ou Bartolomeo di San Concordio, auteur d'une importante somme de confessions, la Summa Pisanella, et promoteur, entre autres, du développement des Arts de la mémoire. Les traités de Bartolomeo et de Cavalca, les sermons de Giordano illustrent une intense réflexion sur le péché, sur la confession et surtout sur les conditions de l'efficacité du discours moral : comment « faire croire » ceux qui vivent dans l'insouciance ? Comment rendre plus impérieux le recours à la confession? Or, les aspects les plus novateurs de l'oeuvre de Buffalmaco paraissent bien s'inscrire dans le prolongement de telles préoccupations. Les fresques illustrent le même souci de diffusion et de vulgarisation de la culture religieuse, témoignent du même effort de perfectionnement des techniques de persuasion et de mémorisation. La multiplication des inscriptions et des adresses au spectateur vise à développer la conscience d'un moi pêcheur, tandis que la diversification des catégories pénales permet de mieux couvrir le champ des fautes, pour mieux capturer chaque fidèle, pour l'inviter à se sentir personnellement visé par la menace de la damnation.

Comme dans la prédication de Giordano da Pisa, l'enfer de Buffalmaco est intégré dans un système ternaire, en interaction avec le thème de la mort et avec celui de la confession : ici, le triomphe de la mort accompagne celui de l'enfer, et plutôt que de repérer dans le cycle l'émergence d'un sens laïcisé de la mort, on préfère y voir une utilisation morale de la mort, dans la tradition du contemptus mundi : la proximité de la mort rappelle l'urgence de la conversion, car après cette vie il sera trop tard pour s'amender. Par ailleurs, l'enfer apparait essentiellement comme une incitation à la pénitence et à la confession, qui est le principal moyen offert par l'Église pour opérer un changement de cap : c'est grâce à elle que les pêcheurs peuvent échapper à l'enfer qui les menace. Ainsi, la fresque du Camposanto intègre l'enfer dans un système d'acculturation religieuse, dont les textes dominicains permettent d'entrevoir les germes et les fondements. Mais il est remarquable de constater que c'est l'image - preuve de sa force et de sa spécificité - qui porte cette exigence à un niveau de cohérence sans équivalent.

\section{Diffusion et perfectionnement du système suppliciant}

L'examen des fresques italiennes entre 1340 et la fin du xve siècle, confirme l'importance de la rupture introduite au campo Santo. Sans doute, le modèle ancien du Jugement et de l'enfer fait-il preuve d'une certaine résistance, en particulier dans les régions septentrionales et dans les milieux plus traditionnels comme ceux du monachisme bénédictin (Pomposa, Viboldone, Lentate, Vérone), tandis que certaines oeuvres, tout en développant les supplices et le principe d'une adaptation à la faute punie, n'adoptent pas de strict compartimentage (Campione d'Italia) ou préfèrent mettre en scène les châtiments des métiers plutôt que ceux des pêchés capitaux (Santa Maria dei Bisognosi). 

diffusion des caractères iconographiques introduits par Buffalmaco. On les retrouve à Florence où il faut toutefois compter davantage avec l'influence de Dante (Bargello, Santa Croce, Santa Maria Novella). On repère également de nombreuses réutilisations explicites de l'enfer pisan, en particulier à San Petronio de Bologne (c. 1410) et dans deux panneaux du Jugement dernier peints par Fra Angelico (lui aussi dominicain). Mais le phénomène principal est le perfectionnement du modèle pisan : ainsi, au Duomo de San Giminiano par exemple (c. 1393-1403), le compartimentage se fait plus rigide; le septénaire est utilisé dans sa totalité et à l'exclusion de toute autre catégorie; la généralisation des souscatégories rend plus concret le système des péchés capitaux; la rigueur de l'adaptation des peines aux fautes, et donc la lisibilité de celles-ci s'en trouve encore renforcée, tout comme la suggestion d'une identification du spectateur avec les damnés.

Enfin, dans les trois dernières décennies $\mathrm{du} \mathrm{xv}^{\mathrm{e}}$ siècle, une nouvelle mutation importante se produit, non plus dans les oeuvres des peintres majeurs de l'époque (qui, en dépit d'exceptions remarquables, comme Signorelli ou plus tard Michel-Ange, délaissent de plus en plus le thème du Jugement dernier et de l'enfer), mais dans les églises rurales du Piémont et les sanctuaires ligures, décorés par des peintres locaux, obscurs mais néanmoins maîtres d'un art vigoureux et plein de ressources. Ainsi, les frères Biazaci témoignent, à Albenga et à Montegrazie (1483), d'une nouvelle affirmation du thème infernal. Cette fois, ce sont les aspects judiciaires (le Christ au centre de la Jérusalem céleste) qui sont relégués dans un angle de la composition, dont les deux-tiers sont occupés par les lieux infernaux, soit une complète inversion du rapport traditionnel entre enfer et Jugement. La mise en ordre de l'enfer, entièrement fondée sur le septénaire et excluant toute représentation de Satan, la multiplication des catégories pénales et la violence des supplices, parviennent à un degré de raffinement supplémentaire.

Mais ici, il ne s'agit plus du Jugement dernier mais d'une représentation systématique de l'autre monde (y compris le purgatoire et le limbe des enfants), dans le temps qui sépare la mort du Jugement dernier. Non que la représentation du jugement des âmes au lendemain de la mort soit une nouveauté, ni même qu'il faille en conclure à l'effacement définitif du Jugement dernier (à quelques kilomètres, en particulier à La Brigue, Canavesio continue de peindre de grandioses Jugements derniers) : simplement, il s'agit de tirer les conséquences de l'affirmation d'un accès immédiat à l'enfer ou au paradis, de souligner toujours plus clairement le rôle de la mort comme pivot de la figure de l'inversion, de faire une place au purgatoire et, plus largement, de figurer l'autre monde dans ses interactions avec le monde des vivants et non tel qu'il sera lorsque celui-ci aura cessé d'exister.

\section{En France : un développement plus quantitatif que qualitatif}

Dans le domaine français, les années 1330-1340 ne correspondent à aucune rupture significative, de sorte qu'on constate, à partir de ce moment, un important décalage avec l'Italie. En effet, il faut attendre un siècle et demi pour trouver, dans les manuscrits de la Cité de Dieu (à partir de 1470), et surtout à Sainte-Cécile d'Albi (1493-1503), une représentation de l'enfer qui puisse être comparée, par son ampleur et par sa structuration, aux grandes fresques italiennes. Et encore l'enfer d'Albi reste-t-il alors très 
largement intégré au Jugement dernier. Les scènes infernales s'inspirent étroitement d'une tradition littéraire, celle de la Vision de Lazare, probablement par le relais des éditions imprimées du Calendrier des bergers. De fait, la figuration de l'enfer trahit par de nombreux indices la dépendance à l'égard d'une tradition issue du livre, ce qui souligne encore la différence avec la situation italienne.

Certes, dès le XIV ${ }^{e}$ siècle, la poussée du thème infernal se fait sentir. En témoigne sa présence croissante dans les Livres d'Heures, lieu mental pourtant consacré à la piété et au recueillement individuels, ou encore son affirmation comme sujet autonome auquel l'enluminure consacre parfois une page entière (en dehors d'une séquence du Jugement dernier). De même, les représentations de l'enfer tendent, dans les fresques du $\mathrm{Xv}^{\mathrm{e}}$ siècle, à occuper une surface croissante. Toutefois, aussi bien dans l'art monumental que dans l'enluminure, la gueule de Léviathan demeure le motif dominant, en dépit d'un processus de marginalisation amorcé au début $\mathrm{du} \mathrm{xv}^{\mathrm{e}}$ siècle. Le développement des supplices reste limité et les rares oeuvres qui en montrent une diversification significative (comme à Kernascleden, fin $\mathrm{xv}^{\mathrm{e}}$ siècle) ignorent toute organisation de l'enfer et (autre différence étonnante avec l'Italie) ne font aucun recours aux inscriptions.

31 Ce décalage surprenant n'est pas seulement le fait de l'image, de sorte qu'on ne peut se contenter de le rapprocher de l'avance dont l'art italien fait preuve dans tous les domaines, ni d'indiquer le développement plus intense de l'art de la fresque, le plus propice à des représentations didactiques de grande ampleur. Ainsi, dans le théâtre religieux, l'évocation développée et ordonnée des châtiments infernaux, l'utilisation du septénaire apparaissent dans une laude italienne du second quart du XIV siècle, L' anticristo e il Giudizio finale, alors que les mêmes transformations ne se font jour de l'autre côté des Alpes qu'avec le Jutgamen général, drame provençal postérieur à 1481. Dans la littérature des visions, la mise en oeuvre d'un système compartimenté (sur la base d'un septénaire imparfait), le développement de la rigueur du système pénal et l'abandon du motif de l'indicibilité de l'enfer caractérisent la Voie d'Enfer et de paradis de Jean de La Motte (c. 1340) et plus encore le Pèlerinage de l'âme de Guillaume de Diguleville (1355-1358). Le décalage avec l'Italie est donc ici moins marqué, même s'il faut remarquer que l'oeuvre de Dante, antérieure de plusieurs décennies, propose d'emblée un ordonnancement plus rigoureux et transforme plus radicalement les modalités d'accès à la révélation de l'autre monde.

\section{De l'enfer comme métaphore à sa tentative de description}

Certes, il ne s'agit pas de faire des Alpes une barrière absolue, d'autant plus que les variations régionales, de part et d'autre, en atténuent la valeur. Reste que le décalage constaté souligne l'existence d'une diversité des représentations infernales, dotées d'une histoire et d'une géographie dont il convient de faire apparaître les rythmes et les nuances. Mais il importe plus encore de formaliser (de schématiser) le mouvement global dont témoignent les oeuvres analysées. Aux XI ${ }^{\mathrm{e}}$-XIII ${ }^{\mathrm{e}}$ siècles, l'enfer est évoqué soit par le recours à la métaphore de l'animalité dévorante (la gueule de Léviathan en France), soit par la figuration d'un lieu largement indistinct (à l'exception de la figure de Satan en Italie), marqué par l'entassement confus des corps, auquel les sinuosités des flammes et des serpents confèrent son caractère instable et inquiétant. Dans les deux cas, l'intérêt se 
porte moins sur la figuration du destin des damnés que sur l'existence de l'enfer comme réalité globale. L'enfer existe comme puissance hostile plus qu'il n'est décrit comme lieu de supplices. Son traitement iconographique peut être comparé à la profusion de superlatifs, à l'énoncé répétitif d'une horreur radicale qui caractérise ses mentions dans la littérature morale; comme elle, l'usage avoue son impuissance à cerner le lieu de l'impensable et ses évocations de l'enfer peuvent être qualifiées de poétique, rythmique ou métaphorique.

33 A partir de la première moitié du xIV siècle, l'attention se fixe au contraire sur le destin des damnés, comme personnes ou comme types, sur l'enfer comme lieu ou comme ensemble de lieux, sur la description d'un système pénal. Sans que l'enfer cesse pour autant d'être un au-delà de tout discours, le discours sur l'enfer, en parole ou en image, se donne comme une révélation complète de l'au-delà. Toujours discours en figure (un acteur le dit à la fin de la représentation du Jutgamen général : « ceci n'est qu'une figure »), l'enfer se donne néanmoins comme une description complète, cherche à éliminer les marques de son infigurabilité. Sans doute sa fonction a-t-elle alors profondément changé et l'on peut se demander si l'enfer n'est pas devenu surtout l'occasion d'un discours moral sur le péché. C'est moins alors le royaume de Satan qui est l'objet de l'image que les fautes que les fidèles sont invités à déchiffrer à travers l'horreur des supplices, à reconnaître en eux-mêmes, et à avouer dans la confession. L'enfer, tel qu'on le voit se mettre en place à Pise est davantage qu'un écho des techniques de l'Art de la mémoire : il est un miroir qui renvoie au sujet l'image d'un moi coupable; il fonctionne comme incitation à la confession, comme fondement d'un recours à la médiation ecclésiale.

\section{Quelle efficacité pour l'enfer?}

Pourtant, il ne suffit pas d'analyser le discours moral auquel participent l'enfer et son image. Encore faut-il s'interroger sur son efficacité. Mais comment savoir si la menace infernale a véritablement eu prise sur les consciences? Plutôt que d'entreprendre une hasardeuse enquête sur la psychologie de l'homme médiéval, on peut s'attacher à des documents peu nombreux mais néanmoins révélateurs, qui suggèrent une conception de l'autre monde moins rigoureuse que celle que l'Église s'emploie à propager. Le thème de la Descente aux limbes peut ainsi être l'occasion d'une évocation de la défaite de Satan et d'une libération complète des âmes, dépassant la portée de l'intervention du Christ telle que la doctrine la définit. Au reste la confusion est entretenue par l'image, qui représente souvent le limbe des patriarches par une gueule de Léviathan, comme s'il s'agissait de l'enfer lui-même. Des textes comme la Visio Anselli (première moitié du $\mathrm{xI}^{\mathrm{e}}$ siècle) évoquent une répétition annuelle de la Descente du Christ, venant libérer les âmes des chrétiens retenues dans les lieux infernaux.

35 À la différence du purgatoire, ces récits ou ces images évoquent une libération acquise non de façon régulière, au terme d'un processus de purification, mais par l'effet d'une faveur exceptionnelle, consentie par les saints ou par la Vierge, et de surcroit souvent présentée comme une entorse à la règle (tympan d'Anzy-le-duc). De telles conceptions sont à la limite de l'orthodoxie, comme en témoigne l'embarras des clercs qui les transmettent tout en cherchant à leur donner une forme plus convenable. Ils sont en cela aidés, à partir du XIII ${ }^{\mathrm{e}}$ siècle, par le purgatoire. Pourtant il est remarquable de constater que, même après son invention, certaines traditions (comme la légende de Trajan ou celle de la Damnation de Dagobert) continuent d'évoquer une libération de l'enfer, qui n'est 
donc pas toujours le royaume de non-retour défini par l'orthodoxie. La possibilité de bénéficier d'une sorte de "droit de grâce ", d'une intervention miraculeuse, ou encore d'inverser le cours de la justice en invoquant des actes de piété aussi modestes que la récitation quotidienne de l'Ave Maria (Voie d'enfer et de paradis de J. de la Motte); tout cela suggère un système beaucoup moins rigoureux, marqué par des tendances miséricordieuses que, déjà, Augustin s'était employé à récuser, et qui limitent d'autant la crainte de l'enfer et l'efficacité du système moral mis en place par l'Église.

Les clercs en témoignent explicitement, en se plaignant souvent du fait que les fidèles ne craignent pas l'enfer. Sans doute faut-il faire la part du topos et de la rhétorique morale. Mais lorsqu'un prédicateur comme Giordano da Pisa développe une analyse détaillée et fine des degrés de croyance à l'enfer et des modalités de son efficacité (et surtout de son inefficacité), il semble qu'il évoque un problème auquel il était réellement confronté. Au reste, le peu que nous savons des conceptions folkloriques de l'autre monde semble leur donner raison. Ainsi, en dépit de leurs spécificités, les témoignages des habitants de Montaillou suggèrent bien l'existence - qu'on devine plus qu'on ne peut véritablement mesurer sa permanence- d'une vision d'un monde des morts qui, sans ignorer complètement toute distinction morale, ne connait que des peines transitoires et qui, au stade de christianisation saisi à Montaillou, réserve l'enfer éternel aux diables, à Judas et aux juifs.

Il faudrait également évoquer le retournement parodique dont le thème infernal peut faire l'objet, ainsi que sa dimension carnavalesque (de Raoul de Houdenc à Rabelais). Mais les remarques qui précèdent suffisent à introduire une complexité qui justifie l'interrogation sur les limites de la peur de l'enfer. Car le développement d'une pastorale et d'une iconographie culpabilisatrices ne peut être compris sans tenir compte de la nécessité de contrecarrer les conceptions folkloriques de l'au-delà, le laxisme miséricordieux, et notamment la confiance dans le pouvoir absolu des intercesseurs. Enfin, au sein même du système chrétien, la menace de l'enfer n'est jamais une fin en soi ; elle est faite pour être dépassée (et à l'exception de ceux qui sont atteints de la «maladie du scrupule », elle l'est sans difficulté). L'objet de l'enfer est moins de "faire peur » que de "faire agir » (et surtout de faire avouer), de façon à se libérer de sa menace. La peur est un composé instable qui, à peine formé, tend déjà vers un autre état. Aussi est-on tenté de parler, plus que d'un «christianisme de la peur », d'un « christianisme du salut obligatoire ».

Encore faut-il se garder de considérer l'enfer uniquement comme une arme manipulée par l'Église pour consolider son pouvoir sur la société. En effet, à opposer ceux qui brandissent la menace sans y être soumis (les clercs) et ceux que l'on oblige à avoir peur (les laïcs), on retomberait dans une conception instrumentale de la religion, assurément appauvrissante. Car bien souvent la menace de l'enfer apparaît, chez les clercs, à usage interne, tandis qu'ailleurs les laïcs eux-mêmes jouent le rôle de relais du faire peur. Le recours à l'image de l'enfer, en particulier lorsqu'elle est introduite dans les Livres d'Heures, répond aussi à un désir de "se faire peur». Enfin, l'efficacité de la menace infernale suppose une prédisposition à la peur (comme le remarque Nietzsche, « ce furent les consciencieux, non les gens sans conscience, qui eurent terriblement à souffrir sous le poids des sermons de pénitence et des angoisses de l'enfer », Aurore, 53). 


\section{L'enfer, discours social sur les pulsions}

39 La question est alors : pourquoi a-t-on peur? Pour tenter d'approcher la résonance des images infernales, la psychanalyse peut inspirer une première démarche. Car, en dépit des problèmes que soulève une utilisation historique des concepts psychanalytiques (et il convient de les affronter), il est difficile de ne pas reconnaître la charge fantasmatique accumulée dans les représentations infernales. Ainsi, la gueule de Léviathan désigne l'enfer tout entier comme un fantasme de dévoration de type cannibalique, tandis que les nombreux aspects qui évoquent un monde ténébreux, une visée de l'intériorité corporelle ou une mise en pièce du corps peuvent être référés aux manifestations du sadisme infantile décrites en particulier par M. Klein. Dans leur ensemble, les images infernales semblent émerger des couches les plus archaïques de la vie psychique.

Toutefois, l'objet de cette démarche n'est pas de parvenir à un diagnostic, en étiquetant les fantasmes cachés derrière les images. Et plutôt que de considérer l'image comme l'énoncé d'un texte pulsionnel, on a intérêt ici à y voir le support des projections multiformes opérées par ses destinataires. L'image infernale apparaît alors comme une formation fantasmatique polyvalente, dont l'analyse ne saurait épuiser la richesse, ni rendre compte entièrement de l'élasticité. En outre, le recours à la psychanalyse vise surtout à étayer une réflexion sur les résonances possibles de l'image infernale et sur les mécanismes (potentiels) de sa réception. En première approximation, on pourrait dire que l'enfer est terrifiant parce qu'il présente au sujet l'image de ses propres pulsions destructrices et qu'il réactive la culpabilité et la crainte du châtiment qui leur sont liées. Mais d'autres effets sont possibles, depuis l'effet terrorisant jusqu'à sa subversion et son retournement jubilatoire, en passant par un effet cathartique qui, en autorisant une expression contrôlée des pulsions sadiques, libère partiellement l'individu d'une part de leur présence persécutrice.

\section{L'enfer comme justice imaginaire et ses rapports avec la justice terrestre}

41 La justice terrestre constitue, pour l'enfer, un autre référent pertinent, d'autant que l'essor du supplice s'y opère simultanément, dans la première moitié du XIV siècle. La mise en place d'un système pénal rigoureux, fondé sur une classification des délits, épaulé par un arsenal suppliciant inscrivant sur le corps des condamnés la marque de la Justice et de sa légitimité fait écho à la " politique de l'effroi » suscitée par l'affirmation de l'État, telle que les historiens l'ont étudiée, à la suite de M. Foucault. De nombreux rapprochements peuvent être établis entre les codes utilisés par les deux justices : usages des mêmes supplices (pendaison d'abord, amputation, démembrement du corps, mais aussi ébouillantement, écorchage, usage de la scie, gavage de crapaux...), recours aux mêmes logiques permettant de lier crime et châtiment. Toutefois, l'identité des deux justices est loin d'être complète et l'enfer conserve une part de spécificité liée à son caractère de justice imaginaire.

Plus profondément, ce sont les relations entre ces deux justices qu'il faut définir. Car ces rapports sont à double sens : la justice terrestre apparait comme un modèle, qui fournit à l'enfer l'image efficace de ses spectacles punitifs ; à l'inverse, de nombreux témoignages 
suggèrent que l'enfer participe à une légitimation de la justice terrestre, dans la mesure où il montre une équivalence, intégré dans le plan de la Providence divine, des châtiments ordonnés par les juges des cours terrestres. De ce point de vue, l'enfer apparait comme une pénalité exemplaire, intégrée dans une justice sans faille, à laquelle nul ne peut échapper (contrairement à ce qui se passe sur terre); il appartient ainsi à un idéal de justice, celui peut-être d'un État dont le souverain serait dans son domaine aussi omnipotent que Dieu dans le sien.

\section{L'enfer. L'individu. La loi. L'État} période considérée. Plus qu'à un simple essor quantitatif, on assiste à la constitution progressive d'un système judiciaire structuré et totalisant. Echo du spectacle des justices terrestres, signe d'une entreprise culpabilisatrice qui, à défaut d'imposer un christianisme de la peur, s'emploie à développer les rudiments d'une morale de l'inversion pour mieux susciter le recours à la médiation ecclésiale, cette mutation doit encore être située au cœur d'une progressive révélation de l'autre monde, qui témoigne d'une mutation des rapports entre l'ici-bas et l'au-delà. Parce qu'il souligne, dans le message chrétien, la contradictoire proclamation de la haine, l'enfer est le signe tragique d'une déchirure entre l'ici-bas et l'au-delà, d'un écart irrémédiable entre le désordre du monde et l'absolue justice de Dieu. Consolation des malheureux, vengeance des persécutés, l'enfer est la protestation des hommes face à l'éloignement de Dieu.

Pourtant, les interactions entre l'enfer et la Justice terrestre, ses utilisations comme rouage d'un système pastoral conçu au bénéfice de l'institution, sa réduction à une grille inspirée par la pratique même de la confession, tout cela traduit en même temps un réseau d'imbrication entre les réalités de l'autre monde et celles de l'ici-bas. Au reste, la configuration infernale inaugurée au début $\mathrm{du}_{\mathrm{XIV}}^{\mathrm{e}}$ siècle ne tarde pas à être contestée (avant de se défaire), puisque Luther refuse le principe d'un compartimentage de l'enfer, condamne, à la suite de Jean Hus, la manipulation cléricale de la menace infernale et réaffirme l'infigurabilité de l'autre monde. Au total, les $\mathrm{XIV}^{\mathrm{e}}-\mathrm{XV}^{\mathrm{e}}$ siècles apparaissent comme un entre-deux marqué par l'équilibre de plusieurs dynamiques contradictoires : moment unique où l'ici-bas et l'au-delà sont comme les deux faces d'une même médaille, ni fondus ensemble, ni totalement séparés. L'au-delà est alors assez distant pour être conçu comme une réalité autonome, mais assez proche pour s'inscrire dans une forme accessible aux hommes. Tel est, dans cet intervalle, le moment de plus grande visibilité de l'enfer. Durant cette période, l'enfer apparait, plus que jamais, au coeur du jeu de la Loi et du Châtiment: écho du grand spectacle des châtiments terrestres autant que des fantasmes destructeurs retournés en crainte d'être détruit, il se fonde, comme eux, sur une potentielle inculpation de l'individu. Et qui sait s'il ne joue pas son rôle dans le passage de la terreur de la pulsion à la crainte du pouvoir : n'est-ce point par lui que s'organise l'échange du pardon et de la soumission? On peut en effet se demander si l'aveu confessionnel, suscité par le choc de l'évocation infernale, n'est pas davantage que la reconnaissance des pêchés identifiés à travers la grille du septénaire. N'est-ce pas en effet une culpabilité bien plus radicale, liée au retour des pulsions agitées par l'enfer, que le chrétien vient avouer à son confesseur?

En tout cas, au moment où l'Église est engagée dans un processus de contrôle accru du corps social et d'encadrement des croyances folkloriques, l'enfer témoigne d'une 
intégration croissante des pulsions destructrices au sein du champ religieux. Elles se résorbent-ou du moins s'expriment-dans les différentes formes de pratiques expiatoires suscités par l'enfer, qui allient toujours la reconnaissance de l'offense faite à Dieu et le moyen de s'acquitter de la dette ainsi contractée. L'enfer autorise ainsi une réorientation des forces pulsionnelles : elles sont l'objet de la transaction par laquelle le sujet échange son pardon contre l'énoncé d'une croyance (la sienne) et la reconnaissance d'un pouvoir (celui de l'Église et, dans une certaine mesure, de l'État qui glisse ici le décalque de sa Loi).

\section{NOTES}

1. NDLR : Une page manquante dans le numéro papier n'a pas pu être numérisée. 